\title{
Comparison of machine learning methods for crack localization
}

\author{
Helle Hein and Ljubov JaAnuska
}

\begin{abstract}
In this paper, the Haar wavelet discrete transform, the artificial neural networks (ANNs), and the random forests (RFs) are applied to predict the location and severity of a crack in an Euler-Bernoulli cantilever subjected to the transverse free vibration. An extensive investigation into two data collection sets and machine learning methods showed that the depth of a crack is more difficult to predict than its location. The data set of eight natural frequency parameters produces more accurate predictions on the crack depth; meanwhile, the data set of eight Haar wavelet coefficients produces more precise predictions on the crack location. Furthermore, the analysis of the results showed that the ensemble of 50 ANN trained by Bayesian regularization and LevenbergMarquardt algorithms slightly outperforms RF.
\end{abstract}

\section{Literature review}

Cracks appear in structural elements mainly as a result of a manufacturing defect or severe exploitation. In consequence, cracks may present a threat to the whole structure since they change behaviour of the construction. Therefore, the discovery and localization of cracks are essential issues in engineering.

The effects of cracks on the global behaviour of a structure can be modelled as a link element (see [7]). In 1957, Irwin came up with the idea that a crack in an elastic element causes a local flexibility due to the strain energy concentration. Irwin modelled the strain concentration using an equivalent spring. This idea led Papaeconomou and Dimarogonas [16] to the development of a more general factor called the stress intensity factor. This idea is still widely used nowadays.

Received November 1, 2018.

2010 Mathematics Subject Classification. 68T05; 70B10; 70E15; 70E17; 70K25.

Key words and phrases. Euler-Bernoulli beam; free vibration; cracks; Haar wavelet; backpropagation network; random forest.

http://dx.doi.org/10.12697/ACUTM.2019.23.13 
The exact close form solution of the uniform Euler-Bernoulli column with a presence of multiple concentrated cracks was proposed by Caddemi and Cali [4] in 2008 and further developed by Caddemi and Morassi [5] in 2013. Dirac's delta function and a rotational spring were used to model the flexibility due to a crack. Alternatively, an analytical approach using a transfer matrix method was described by Attar [1].

Nandwana and Maiti [15] modelled the transverse vibration of a slender beam in the presence of an inclined edge crack using a rotational spring and the measurement of the natural frequencies. The characteristic equation obtained from the vibration analysis of the beam was manipulated to give a relationship between the stiffness of the spring and the location of the crack. Plots of spring stiffness and crack locations were obtained for the three lowest transverse modes. Through a large number of numerical examples on the inclined edge crack, the beam modelling through the rotational spring was feasible for at least small crack angles. The error in the calculations of the crack location was less than 4.5 per cent.

In the last decades, the inverse problem of crack identification (location and depth) using the changes in the measured frequencies of a cracked element has been in focus. According to [12, 18, 9], vibration parameters are still the best and one of the easily available indicators of the structure's overall technical condition since the natural frequencies can easily be measured, they are less distorted by noise, and a presence of a crack reduces significantly the frequency domain.

By now, the frequencies have usually been measured by acceleration sensors or laser vibrometers either obtained numerically using Galerkin method or Rayleigh-Ritz method. Nonetheless, it is a challenging task to localize cracks in an Euler-Bernoulli beam using some vibration data. In order to obtain trustworthy results, a proper analytical model, a signal analysis, and an efficient numerical technique have to be combined.

Several researchers have tackled the inverse problem using artificial neural networks (ANN). This is due to the fact that ANNs are capable of pattern recognition, classification, signal processing, and system identification. In this paper, it is assumed that the ANNs can be a powerful tool in complementing vibration-based damage detection methods since changes in the dynamic parameters of a structure are attributed to certain parameters of a damage.

In [13], Mahmoud and Kiefa investigated a steel cantilever beam with a single edge crack. The authors used the general regression neural networks (GRNN) and the first six natural frequencies in order to predict the crack size and crack location. The natural frequencies were calculated using Mmatrix technique and Newton-Raphson method. Mahmoud and Kiefa drew several important conclusions: 
- if the crack depth is smaller than $a / h=0.2$, it is difficult to localize the crack since the change in the natural frequencies are small;

- the first two natural frequencies $\left(\omega_{1}, \omega_{2}\right)$ are not enough to predict the depth $a / h$ and location $x / L$ of the crack since the natural frequencies vary in a cyclic manner.

The calculations by GRNN in [13] showed that the network was capable to identify cracks in the range $0.08<a / h \leq 0.82$ quite accurately $\left(R^{2}=0.87\right.$ for the location and $R^{2}=0.97$ for the depth).

He et al. [11] implemented the genetic algorithms for detecting a shaft crack. In the research, the authors formulated the shaft crack detection as an optimization problem by means of finite element method. The accuracy of the crack location prediction was 98.46 per cent and in case of the crack depth prediction - 88.22 per cent.

In 2015, Sutar et al. [21] tried to realize a neural network based controller for the crack identification in an aluminium cantilever. The input parameters to the controller were relative divergence of the first three natural frequencies obtained experimentally and the output parameters were relative crack depth and location. For the training, a three-layered feed-forward back propagation ANN with nine neurons in the hidden layer was used. The results showed that the mean absolute percentage error between the actual and the predicted responses is less than 15 per cent.

Although the results look promising, there are some issues that need to be discussed and resolved. In [23], Worden et al. state that "in engineering problems, data are often the result of expensive experiments and will be in short supply; in this case, the only way to ensure generalisation is to restrict the number of weights in the network". On the other hand, neither of the above-mentioned researchers have used the random forest $(\mathrm{RF})$ as a predictive model nor applied Haar wavelet transform to the mode shape for data generation.

The RF is a classifier defined by Breiman for the classification and regression problems. The statistical method is based on a large set of unpruned decision trees, which are known as classifiers. The method is widely used in economics and bioinformatics. A detailed description of the method can be found in [3].

The Haar wavelet is the first known and yet the simplest wavelet introduced in 1909 by Alfred Haar. The wavelet transforms have advantages over traditional Fourier transforms for representing functions that have discontinuities and sharp peaks, and for accurately deconstructing and reconstructing finite, non-periodic and/or non-stationary signals (see [8]). Due to time-frequency localization, the wavelet transform can reveal some hidden parts of data that other signal analysis techniques fail to detect (see [24]). 
The observation of the wavelet sensibility to non-linear structure changes was supported experimentally by Rucka and Wilde in [19, 20].

In this paper, the Haar wavelet discrete transform, the ANN, and the RF are applied to predict the location and severity of the crack in an EulerBernoulli cantilever subjected to the transverse free vibration. It is assumed that the first mode shape can be applied to the Haar wavelet transformation with a small level of resolution in order to find appropriate predictors for the machine learning methods. Next, a deep investigation into two data collection sets (the natural frequency parameters based and the Haar wavelet coefficients based) and the machine learning methods are carried out. No related work has been found in literature.

\section{Mathematical model of Euler-Bernoulli beam with an open crack}

According to Rizos et al. [17], a cracked uniform beam can be simulated as two uniform beams joined together by a rotational spring at the crack due to the localized crack effect. The continuity and compatibility conditions at the crack $\left(x=L_{1}\right)$ take the form

$$
\begin{aligned}
& W_{1}=W_{2}, \\
& \frac{d^{2} W_{1}}{d x^{2}}=\frac{d^{2} W_{2}}{d x^{2}}, \\
& \frac{d^{3} W_{1}}{d x^{3}}=\frac{d^{3} W_{2}}{d x^{3}}, \\
& \frac{d W_{1}}{d x}+c \frac{d^{2} W_{1}}{d x^{2}}=\frac{d W_{2}}{d x},
\end{aligned}
$$

where $W_{1}$ and $W_{2}$ are the mode shapes on the left and right beam sections, respectively. The bending constant of the massless spring $c$ is presented in [6] as

$$
c=5.346 \frac{h}{E I} J\left(\frac{a}{h}\right),
$$

where $h$ is the thickness of the beam, $a$ is the depth of the crack, and $J\left(\frac{a}{h}\right)$ is the dimensionless local compliance function proposed by Dimarogonas et al. [6]:

$$
\begin{aligned}
J\left(\frac{a}{h}\right)= & 1.8624\left(\frac{a}{h}\right)^{2}-3.95\left(\frac{a}{h}\right)^{3}+16.375\left(\frac{a}{h}\right)^{4} \\
& -37.226\left(\frac{a}{h}\right)^{5}+76.81\left(\frac{a}{h}\right)^{6}--126.9\left(\frac{a}{h}\right)^{7} \\
& +172\left(\frac{a}{h}\right)^{8}-143.97\left(\frac{a}{h}\right)^{9}+66.56\left(\frac{a}{h}\right)^{10} .
\end{aligned}
$$

This mathematical model is used to calculate the first eight natural frequency parameters. 


\section{Data sets}

An essential prerequisite to train a machine learning model to predict a given phenomenon is to identify a set of features that may serve as indicators of the phenomenon. The literature review shows that most of the studies consider only the first three natural frequencies (rarely the first five natural frequencies) since the calculation of high frequency parameters is complicated and time-consuming. The frequencies are usually calculated using FEM or power series technique and used as the input parameters of a prediction model (see [7]). According to Mahmoud and Kiefa [13], the fewer the number of the natural frequencies, the less accurate the ANN predictions are.

In the present paper, a more rigorous and exhaustive approach has been adopted by inspecting two independent data sets extracted or derived from the modal analysis: the first eight natural frequency parameters and up to 64 Haar wavelet coefficients derived from the first mode shape. It has been assumed that due to the Haar wavelet transform, the prediction model can be built easier and faster than the frequency based model.

In order to evaluate the hypotheses, two sets of data have been calculated numerically and compared to the ones available in literature (see [18]). In the first set, each pattern contains scaled values of the crack depth and location, and the first eight frequency parameters of vibration. In the second set of data, each pattern contains scaled values of the crack depth and location, and 64 Haar wavelet coefficients. The coefficients have been calculated and normalized as following:

- calculate the first natural frequency parameter $k_{1}$ of the cracked cantilever and obtain the Haar transform of the first mode shape $y_{c}$;

- calculate the first natural frequency parameter $k_{1}$ of the intact cantilever and obtain the Haar transform of the first mode shape $y_{i}$;

- calculate the difference $y_{c}-y_{i}$;

- normalize the results.

In general, the calculation of the first eight frequency parameters is more time consuming than the calculation of the 64 Haar wavelet coefficients, but it depends on the mesh used and accuracy required. The computing time for calculating frequency parameters can be reduced substantially by applying higher order methods which allow to achieve higher accuracy with a smaller mesh (see [14]).

In total, each dataset contained 800 patterns. The location and depth of the cracks were selected randomly and the parameters were calculated. The minimum and maximum values of crack depth were set between 0.01 and 0.5. The location values of the cracks were set in the range from 0.001 to 0.99 . The beam length was scaled to 1 . The crack characteristics in the data sets covered the whole range of the beam. 
Before building the prediction models, the correlation between metrics and the observed crack characteristic were calculated (Figure 1).

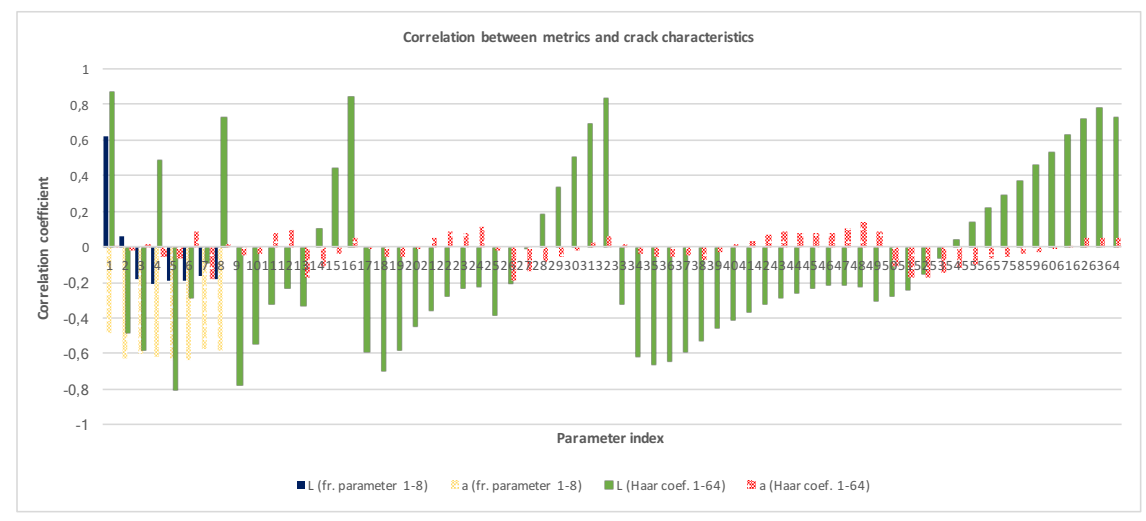

FiguRE 1. Correlation between the crack characteristics and the natural frequency parameters or the Haar wavelet coefficients.

The correlation analysis showed that a few individual metrics from the Haar wavelet data set had a strong positive or negative correlation $(R>$ $|0.7|)$ with the crack location. A moderate negative correlation was observed between a few individual metrics from the frequency parameter data set and the crack depth. The latter was comfirmed by Hakim et al. [10] that the natural frequencies of the first five modes have more influence in predicting the damage identification. In this reseach, there were no individual metrics which strongly correlated with both characteristics of the crack at the same time (Tables 1 and 2). This was in line with findings from other studies ([13]). Thus, it was decided to construct prediction models based on a combination of the variables.

TABLE 1. Correlation between the natural frequency parameters and the crack characteristics ( $L$ - location, $d$ - depth).

\begin{tabular}{l|c|c|c|c|c|c|c|c}
\hline Crack & $f_{1}$ & $f_{2}$ & $f_{3}$ & $f_{4}$ & $f_{5}$ & $f_{6}$ & $f_{7}$ & $f_{8}$ \\
\hline$L$ & 0.621 & 0.055 & -0.178 & -0.210 & -0.190 & -0.191 & -0.167 & -0.181 \\
$d$ & -0.488 & -0.631 & -0.597 & -0.618 & -0.632 & -0.632 & -0.574 & -0.586
\end{tabular}

\section{Feed-forward neural networks with back propagation}

Prediction model building in ANNs refers to selecting the "optimal" network architecture, network topology, data representation, training algorithm, training parameters, and terminating criteria such that some desired level 
TABLE 2. Correlation between the Haar wavelet coeffcients and the crack characteristics $(L$ - location, $d$ - depth).

\begin{tabular}{l|c|c|c|c|c|c|c|c|c}
\hline Crack & $h_{1}$ & $h_{5}$ & $h_{8}$ & $h_{9}$ & $h_{16}$ & $h_{32}$ & $h_{62}$ & $h_{63}$ & $h_{64}$ \\
\hline$L$ & 0.868 & -0.806 & 0.727 & -0.781 & 0.846 & 0.835 & 0.713 & -0.776 & 0.725 \\
$d$ & 0.007 & -0.065 & 0.013 & -0.048 & 0.052 & 0.058 & 0.046 & 0.054 & 0.047
\end{tabular}

of performance is achieved [22]. Therefore, various networks were examined in this research. Each ANN was constructed by fitnet function in the MATLAB environment.

The following five training functions were assessed: Levenberg-Marquardt back propagation (LM), scaled conjugate gradient back propagation (SCG), resilient back propagation algorithm (RP), Broyden-Fletcher-GoldfarbShanno back propagation (BFGS), and Bayesian regularization back propagation (BR). In the RP, the learning rate was set to 0.01 .

In order to avoid the construction of an unreasonably large networks, the number of hidden layers was set to one. According to [2], a network of two layers, where the first layer is sigmoid and the second layer is linear, can be trained to approximate any function arbitrarily well. Following this recommendation, the Elliot sigmoid function was chosen for the hidden layer and the linear transfer function - for the output layer. The Elliot sigmoid function is approximately four times faster than the symmetric sigmoid [2] since it does not use exponents.

The number of neurons on the hidden layer was initially set to ten and gradually increased to 150 with step 20 in order to find a reasonable solution.

The number of training patterns was initially set to 100 and gradually increased to 700 with step 100. Each network was trained 50 times starting at randomly chosen initial conditions in the weight space in the range of -1 to 1 and a random division of the initial data set into training and validation:

- $70 \%$ of the training set has been used for training: computing the gradient and updating the network weights and biases;

- $30 \%$ of the training set has been used for validation to tune the model.

The test set contained 50 patterns which were shown to the networks during the training. The networks assessment was based on this set.

To speed up the learning process [2], the training was performed in the batch mode. The network training was stopped at one of conditions:

(1) the magnitude of the gradient was less than $1 e-7$,

(2) the number of validation checks reached 6 ,

(3) the number of epochs reached 1000 ,

(4) the performance value became 0.00 .

The training time was not limited. 
Table 3 shows the average result of training and testing on the natural frequency parameters for each algorithm. In Table 4, the best result (based on the mean square error of 50 test crack predictions) and the respective network configuration for each algorithm are tabulated. Both tables contain the average training and testing mean square errors, the correlation parameter for the predicted location and depth of the test cracks, the average learning time per network training, the number of neurons on the hidden layer, the number of patterns in the training set, and the reason of training stop according to the list above.

In case of training on the natural frequency parameters, the best average mean square error of 50 trainings to localize 50 cracks was 0.0033 . The average correlation parameter for the location prediction was 0.9765 ; the average correlation parameter for the depth prediction was 0.9713. The result was obtained by the networks with ten neurons on the hidden layer trained by Levenberg-Marquardt algorithm using 700 training patterns with eight natural frequency parameters in each.

The results of the scaled conjugate gradient, resilient propagation, and BFGS Quasi-Newton back propagation algorithms were mutilated by overfitting: the mean square error was higher on the testing set than on the training set.

According to Table 3, the Bayesian regularization showed the second accurate result reaching the maximum number of epochs. An additional test with up to 6000 epochs resulted in significant improvement in prediction accuracy: training $m s e=0.0022$, testing $m s e=0.0022$, crack location correlation_parameter $=0.9745$, and crack depth correlation_parameter $=$ 0.9620. The average time per training increased more than six times.

In case of training on Haar wavelet coefficients, the best average mean square error of 50 trainings was 0.0031 . The average correlation parameter for the location prediction was 0.9998 ; the average correlation parameter for the depth prediction was 0.8389 . The average result was obtained by the network with 10 neurons on the hidden layer. The network was trained by Bayesian regularization algorithm using 300 training patterns and 1000 epochs. In each pattern, there were eight Haar coefficients. An increase of the iteration number to 4500 and 6000 epochs did not show any satisfactory improvement in prediction accuracy.

For the discussed approaches, the influence of the number of neurons and training patterns on the mean square error of 50 crack localization was studied. According to the analysis, the approach based on the natural frequency parameter data set required at least 700 training patterns in order to learn relationship between inputs and outputs. In case of the Haar coefficient data set, 300 training patterns were sufficient. 


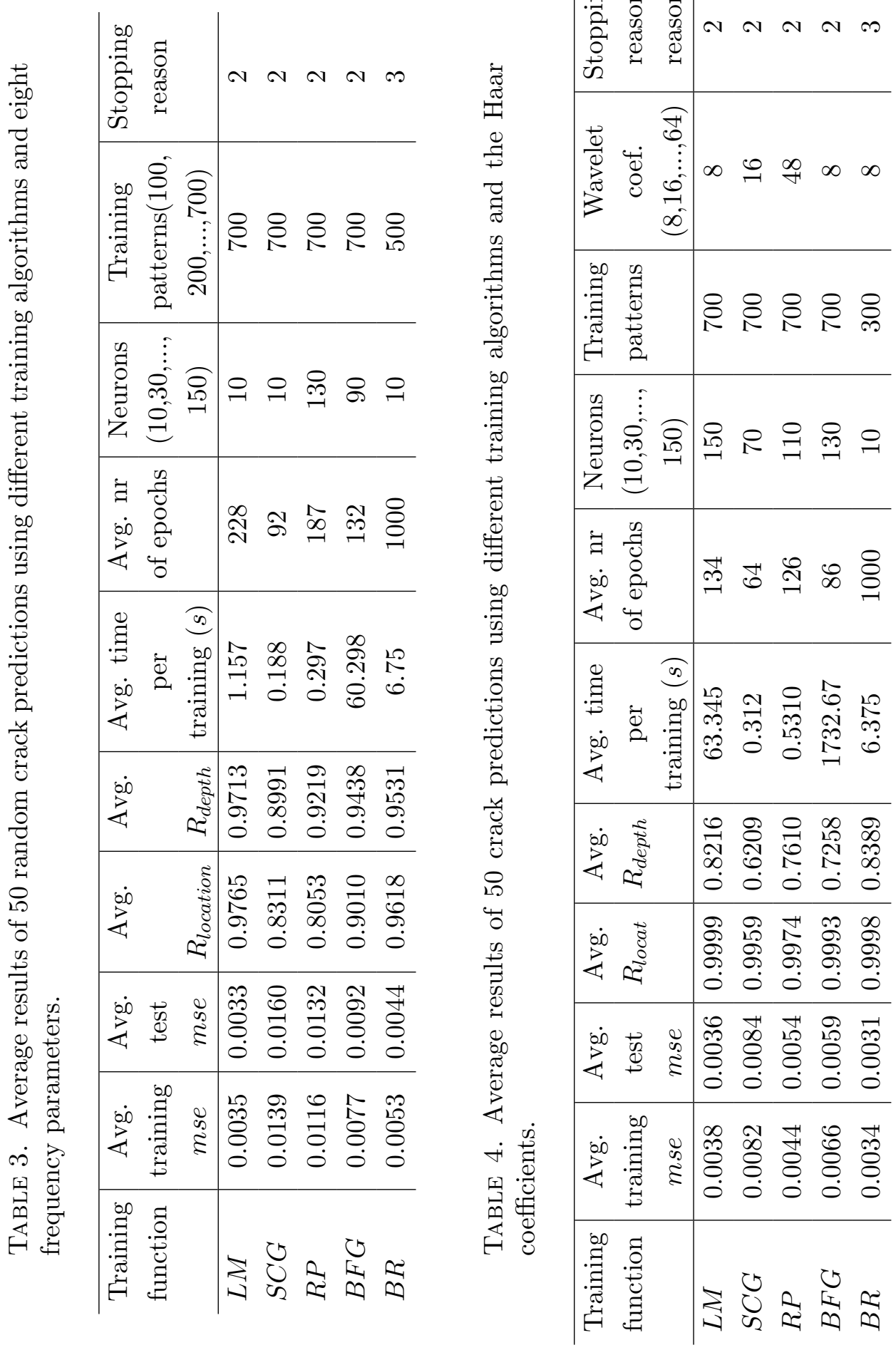


Next, an ensemble of 50 neural networks of the same architecture was proposed to calculate an averaged crack parameters. The idea originated from the articles [10] and [2]. Figure 2a shows the results of 50 networks with ten neurons on the hidden layer trained by the Levenberg-Marquardt algorithm and 700 training patterns containing eight frequency parameters. The testing mse reduced from 0.0033 to 0.0013 . Figure $2 \mathrm{~b}$ shows the results of 50 networks with 10 neurons on the hidden layer trained using the Bayesian regularization and 300 training patterns containing eight Haar coefficients. The testing mse reduced from 0.0031 to 0.0023 .

In Figure 2, it is seen that large errors occur at crack depth identification. The observation is confirmed by the correlation analysis. The regression plots for both characteristics and approaches are shown in Figure 3. In both cases, the correlation parameter $(\mathrm{R}$-value) between the outputs and the crack location targets are very close to 1 , which indicates a good fit and high precision of the networks in crack localization. However, the ensemble of networks trained on Haar wavelets predicts crack location slightly better than the ensemble of networks trained on frequency parameters $(R=0.9999$ and $R=0.9908$, respectively). In case of depth prediction, the results are vice versa. The ensemble of networks trained on frequency parameters predicts crack depth better than the ensemble of networks trained on Haar wavelet coefficients ( $R=0.9772$ and $R=0.8871$, respectively). The observation is in line with the correlation coefficients shown in Tables 1, 2.

The error distributions for both approaches are shown in Figure 4. It shows that in 98 per cent cases, the crack location can be predicted with 99 per cent accuracy using the Haar coefficients as inputs; in case of crack depth, the error is nearly 1.5 per cent in 80 per cent cases using the natural frequency parameters as inputs.

In order to improve the previous results, it was decided to combine both methods: an ensemble of 50 networks trained on the natural frequency parameters to predict the crack depths, and an ensemble of 50 networks trained on the Haar coefficients to predict the location of the cracks. In this case, mse $=0.0002$, location correlation_parameter $=1$, and depth correlation_parameter $=0.9868$. The results are shown in Figure 5 .

\section{Random forest}

Next, the crack parameter identification was examined with the aid of the RF. The following configurations of the RFs were examined:

- number of predictors in samples $(\eta)$ : eight natural frequencies, or eight, 16, 32, 48 or 64 Haar coefficients;

- number of predictors without replacement in samples $(p): 1 / 6,1 / 2$ and $2 / 3$ of the predictors in the pattern; 


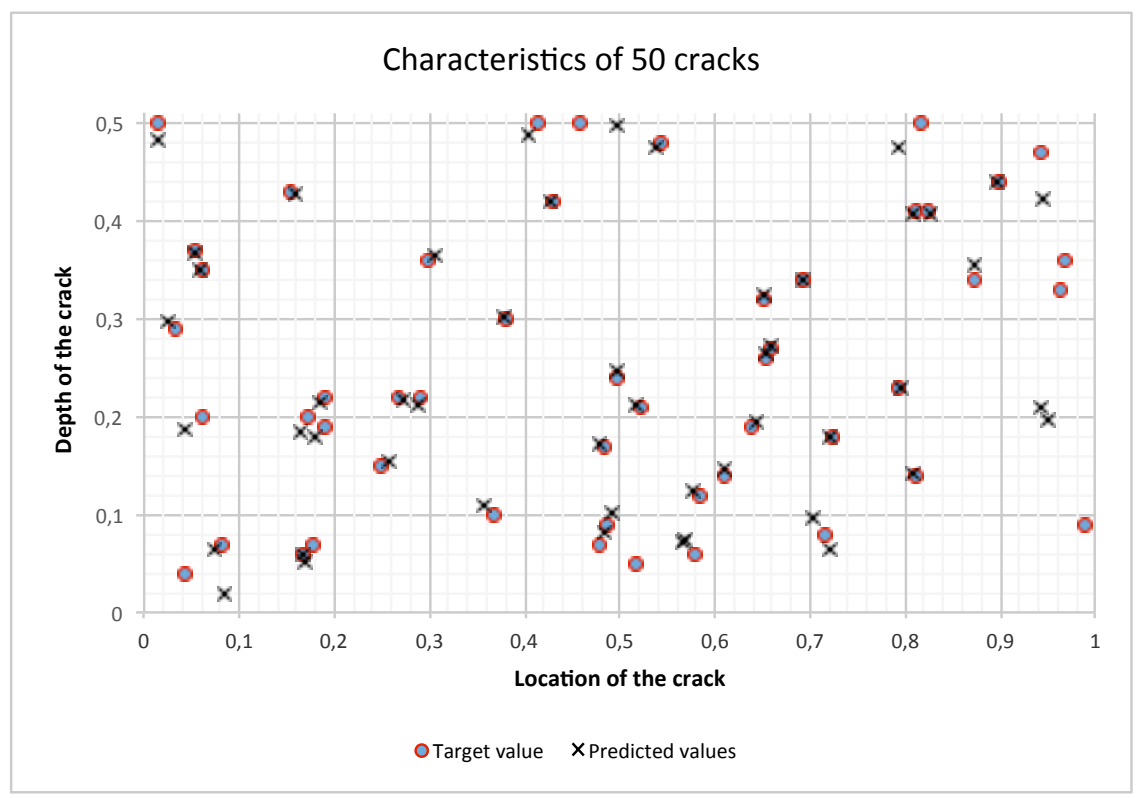

(A) Crack characteristics predicted by an ensemble of 50 ANNs trained by Levenberg-Marquardt algorithm and 600 training patterns containing eight frequency parameters.

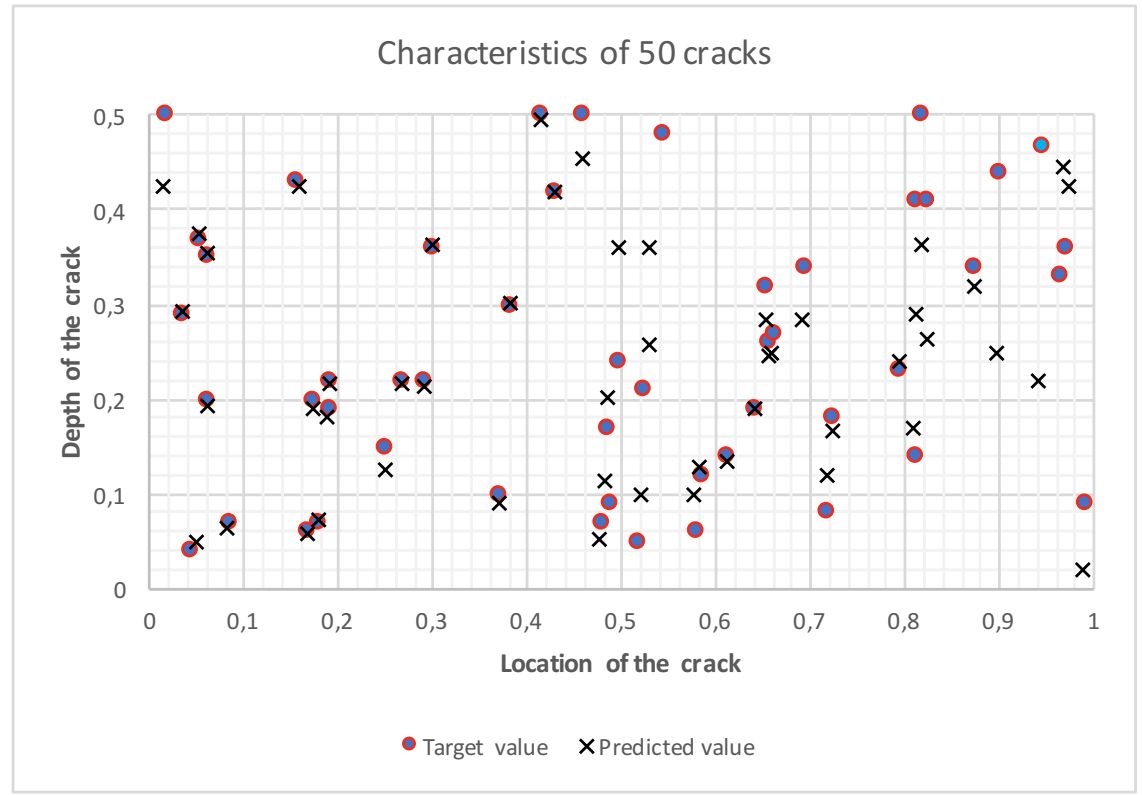

(в) Crack characteristics predicted by an ensemble of 50 ANNs trained by Bayesian regularization and 300 training patterns containing eight Haar coefficients.

FiguRE 2. Characteristics of 50 random cracks: $\mathrm{x}$ - predicted depth and location; o - target values. 


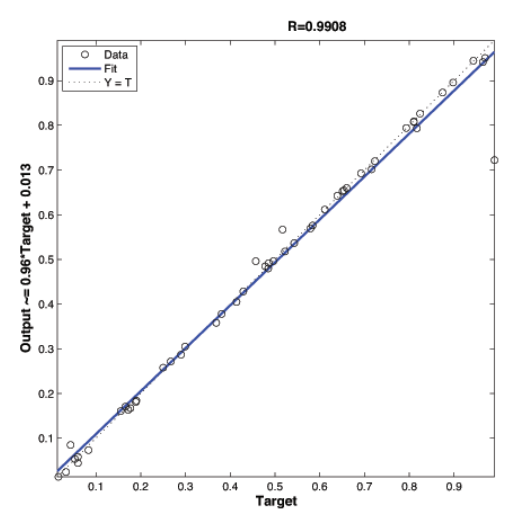

(A) Location prediction.

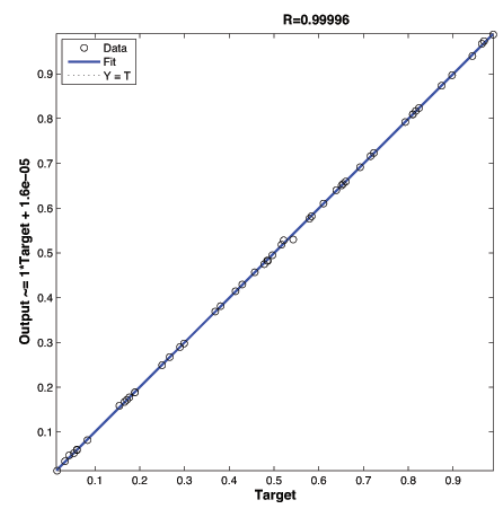

(c) Location prediction.

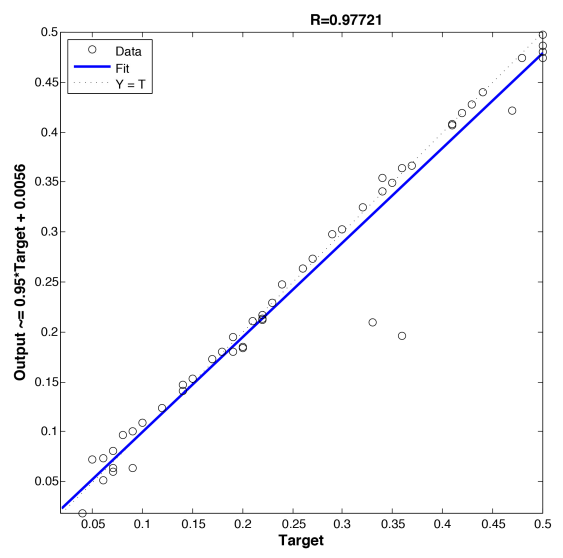

(B) Depth prediction.

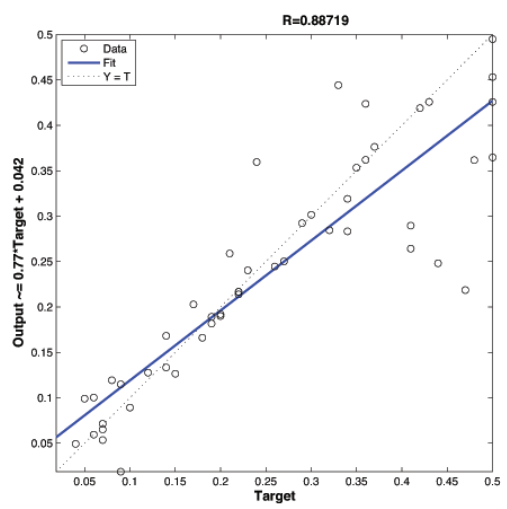

(D) Depth prediction.

FiguRE 3. Regression plots for the predicted characteristics of 50 cracks. (a), (b) predictions are based on eight frequency parameters; (c), (d) predictions are based on eight Haar coefficients.

- number of trees: 10, 20, 30, 40, 50, 75, 100, 150, 200, 250, 300, 400, 500, 750, 1000;

- number of training patterns: $100,200, \ldots, 700$.

The best five average results of 50 runs are shown in Table 5 and Table 6 . In case of training on the natural frequency parameters, the best average mean square error of 50 trainings to localize and estimate the severity of 50 cracks were 0.0020 and 0.0008 , respectively. An average correlation parameter for the location prediction was 0.9896 ; an average correlation parameter 
TABLE 5. The most accurate results of the prediction of 50 crack locations by the RF using different topologies and a data set of the natural frequency parameters $(L$ - location, $D$ - depth of the crack).

\begin{tabular}{|c|c|c|c|c|c|c|c|}
\hline & $\begin{array}{c}\mathrm{Nr} \text { of } \\
\text { training } \\
\text { patterns }\end{array}$ & $\eta$ & $p$ & $\begin{array}{l}\mathrm{Nr} \text { of } \\
\text { trees }\end{array}$ & $\begin{array}{c}\text { Avr. } \\
\text { test } \\
M S E\end{array}$ & $\begin{array}{l}\text { Avg. } \\
R_{\text {locat }}\end{array}$ & $\begin{array}{c}\text { Avg. } \\
\text { training } \\
\text { time }\end{array}$ \\
\hline \multirow[t]{6}{*}{$\mathrm{L}$} & & & & & & & \\
\hline & 500 & 8 & 4 & 20 & 0.0020 & 0.9896 & 0.1108 \\
\hline & 500 & 8 & 6 & 10 & 0.0021 & 0.9885 & 0.0674 \\
\hline & 600 & 8 & 4 & 10 & 0.0021 & 0.9880 & 0.0619 \\
\hline & 500 & 8 & 4 & 40 & 0.0022 & 0.9888 & 0.2112 \\
\hline & 600 & 8 & 4 & 400 & 0.0023 & 0.9881 & 2.1502 \\
\hline \multirow[t]{6}{*}{$\mathrm{D}$} & & & & & & & \\
\hline & 700 & 8 & 6 & 50 & 0.0008 & 0.9841 & 0.3847 \\
\hline & 700 & 8 & 4 & 100 & 0.0008 & 0.9821 & 0.6683 \\
\hline & 600 & 8 & 6 & 300 & 0.0008 & 0.9826 & 2.0682 \\
\hline & 700 & 8 & 6 & 750 & 0.0008 & 0.9820 & 5.7232 \\
\hline & 700 & 8 & 4 & 40 & 0.0009 & 0.9812 & 0.2739 \\
\hline
\end{tabular}

TABLE 6 . The most accurate results of prediction of 50 crack locations by the RF using different topologies and a data set of the Haar wavelet ( $L$ - location, $D$ - depth of the crack).

\begin{tabular}{c|c|c|c|c|c|c|c}
\hline & $\begin{array}{c}\text { Nr of } \\
\text { training } \\
\text { patterns }\end{array}$ & $\eta$ & $p$ & $\begin{array}{c}\text { Nr of } \\
\text { trees }\end{array}$ & $\begin{array}{c}\text { Avr. } \\
\text { test } \\
M S E\end{array}$ & $\begin{array}{c}\text { Avg. } \\
R_{\text {locat }}\end{array}$ & $\begin{array}{c}\text { Avg. } \\
\text { training } \\
\text { time }\end{array}$ \\
\hline $\mathrm{L}$ & & & & & & & \\
& 700 & 8 & 6 & 10 & 0.0000 & 0.9999 & 0.0653 \\
700 & 8 & 6 & 20 & 0.0000 & 0.9999 & 0.1201 \\
& 700 & 8 & 6 & 100 & 0.0000 & 0.9999 & 0.5646 \\
& 500 & 8 & 6 & 10 & 0.0000 & 0.9999 & 0.0587 \\
& 500 & 8 & 6 & 100 & 0.0000 & 0.9999 & 0.4883 \\
\hline $\mathrm{D}$ & & & & & & & \\
& 700 & 16 & 11 & 10 & 0.0061 & 0.8491 & 0.1365 \\
& 64 & 43 & 100 & 0.0063 & 0.8395 & 2.9505 \\
& 700 & 64 & 43 & 150 & 0.0064 & 0.8361 & 4.4674 \\
& 700 & 64 & 32 & 75 & 0.0064 & 0.8374 & 1.7222 \\
& 700 & 64 & 32 & 150 & 0.0064 & 0.8378 & 3.4962
\end{tabular}




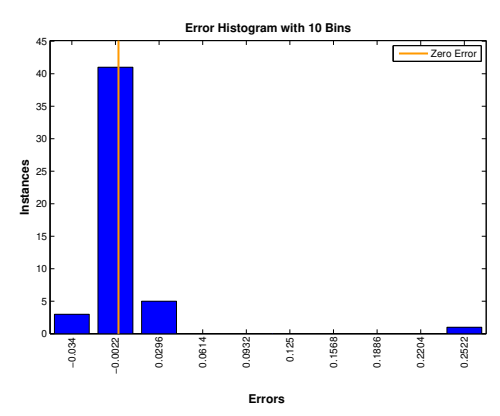

(A) Errors in location prediction.

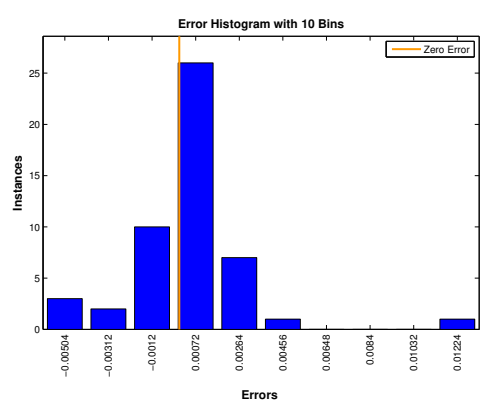

(c) Errors in location prediction.

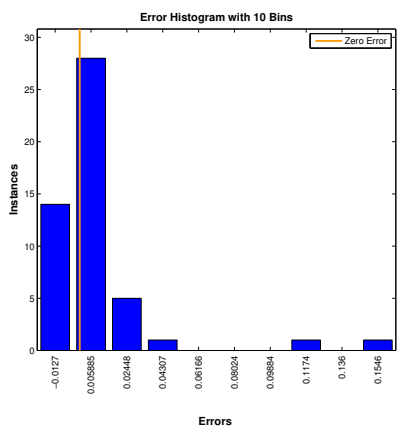

(B) Errors in depth prediction.

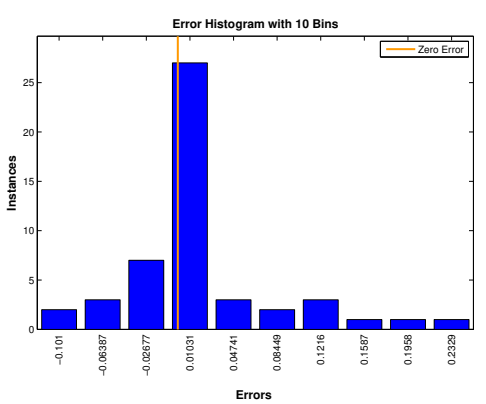

(D) Errors in depth prediction.

FiguRE 4. Error distribution for the predicted characteristics of 50 cracks. (a), (b) predictions are based on eight frequency parameters; (c), (d) predictions are based on eight Haar coefficients.

for the depth prediction was 0.9841 . The results were obtained by the RF with 20 trees, 500 training patterns, and four predictors without replacement and by the RF with 50 trees, 700 training patterns, and six predictors without replacement, respectively.

In case of training on the Haar wavelet coefficients, the best average mean square error of 50 trainings to localize and estimate the severity of the cracks were 0.0000 and 0.0061 , respectively. An average correlation parameter for the location prediction was 0.9999; an average correlation parameter for the depth prediction was 0.8491 . The average result was obtained by the RF with 10 trees using 700 training patterns and six or eleven predictors without replacement.

The error distribution and correlation parameter of a combination of RFs are shown in Figure 6. The mean square error for the combination is 0.0004 . 


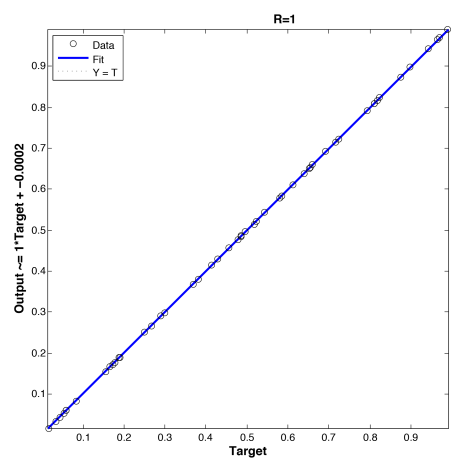

(A) Location prediction.

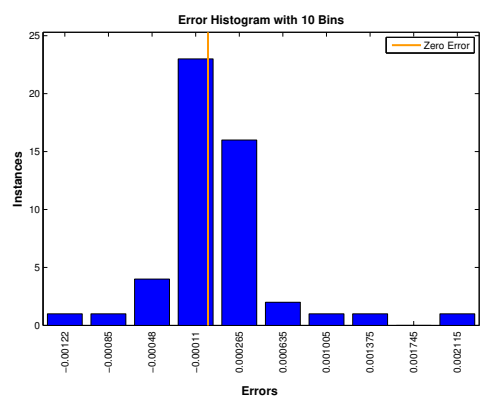

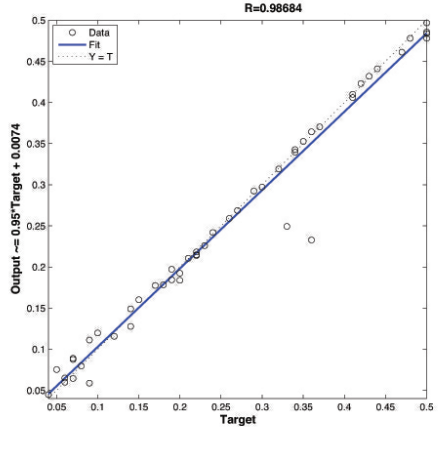

(B) Depth prediction.

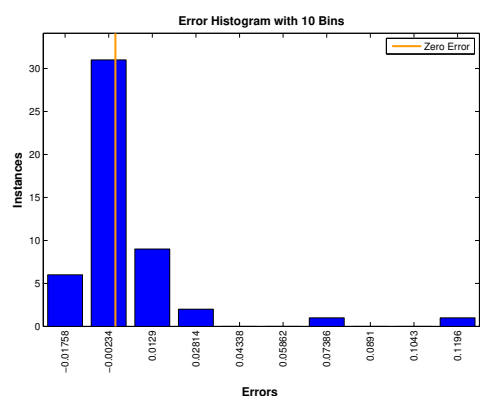

(C) Error distribution in location pre- (D) Error destribution in depth prediction. diction.

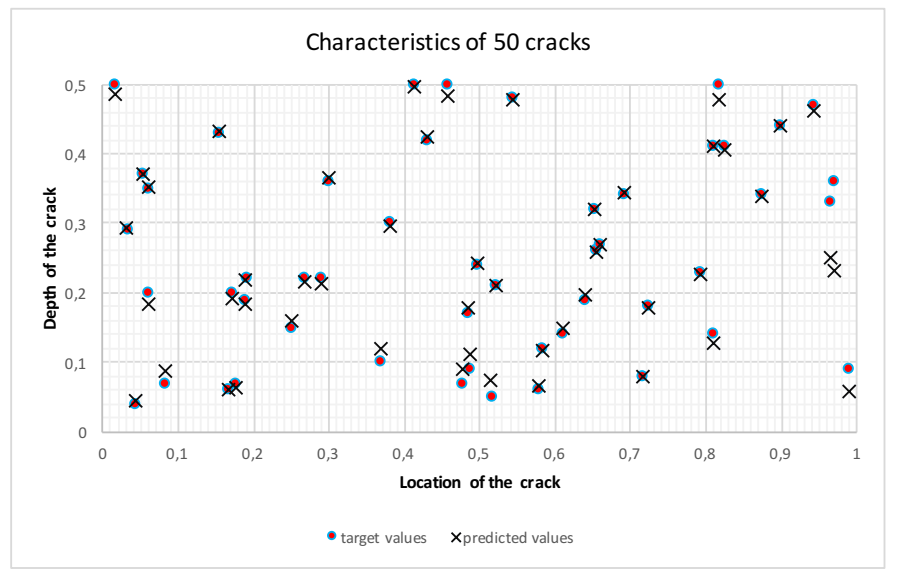

(E) Characteristics of the target and predicted cracks.

FigURE 5. 50 ANNs trained on frequency parameters and Levenberg-Marquardt method to predict depth of the crack; 50 ANNs trained on the Haar coefficients and Bayesian regularization to predict location of the crack. 


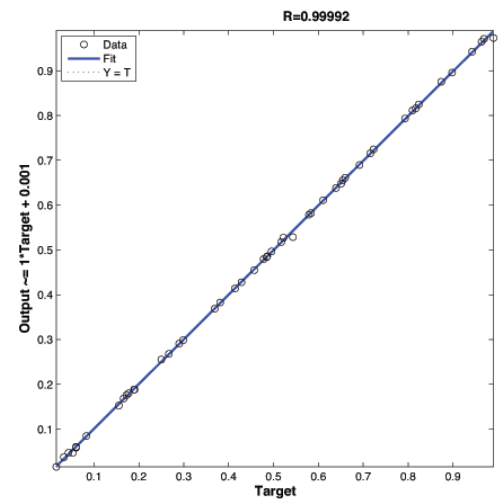

(A) Location prediction.

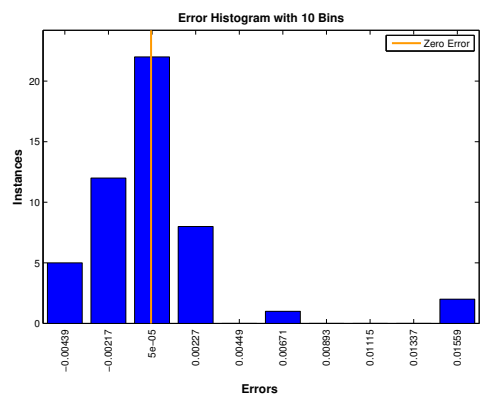

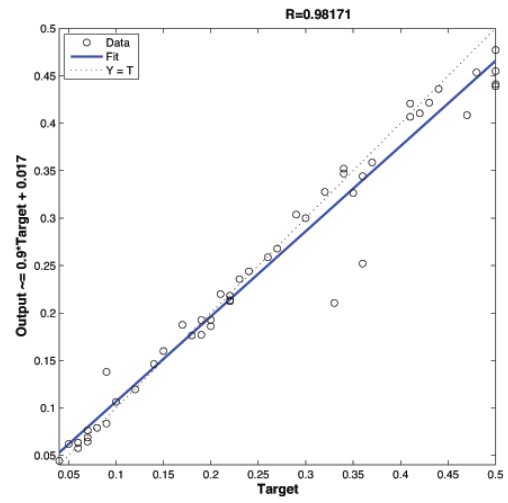

(B) Depth prediction.

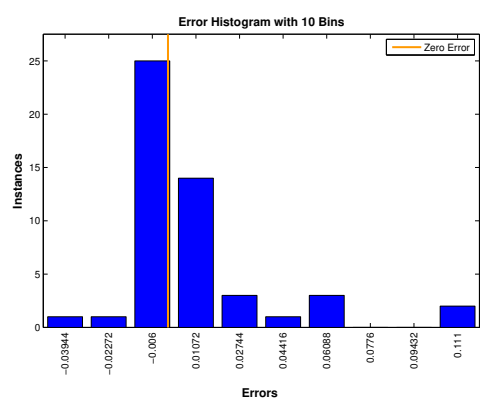

(C) Error distribution in location pre- (D) Error destribution in depth prediction. diction.

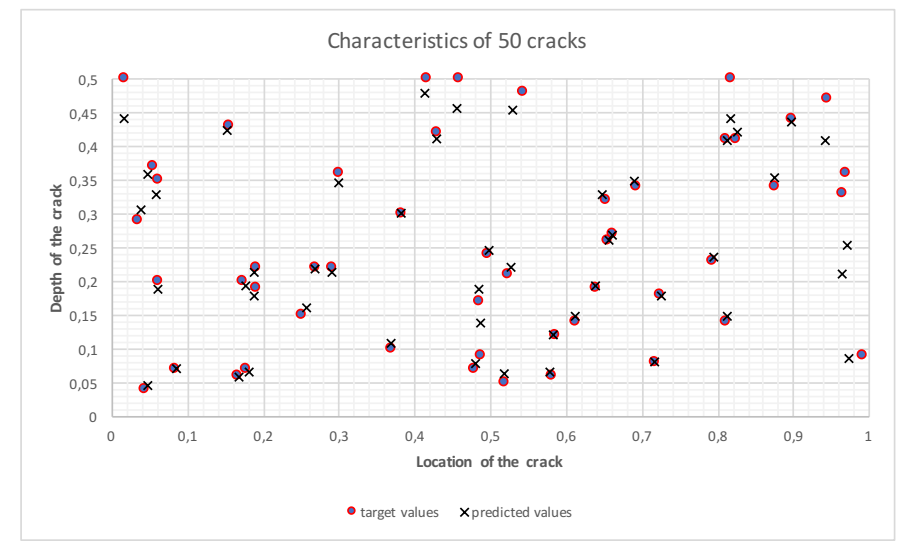

(E) Characteristics of target and predicted cracks.

FiguRE 6. 50 RFs trained on the frequency parameters to predict the depth of the cracks; 50 RFs trained on the Haar coefficients to predict location of the crack. 
As in the case with the ANN, the data set of the natural frequency coefficients produced better predictions on the depth of the cracks; meanwhile, the data set of eight Haar wavelet coefficients produced precise predictions on the crack location.

\section{Discussion and conclusion}

The major aim of the present paper was to find an efficient model for crack localization. A feed-forward back propagation ANN and RF were incorporated for developing a prediction model of the formulations. Five different training algorithms were used to train ANNs. The second objective of the paper was to compare the performance of aforementioned algorithms with regard to prediction accuracy. The third objective of the paper was connected to the nature of the training sets and their size. Two data sets were calculated numerically. The first one contained the first eight natural frequency parameters; the other one - up to 64 Haar wavelet coefficients derived from the first mode shape. The hypotheses were evaluated on the testing set on the bases of the MSE between the output and target values.

The analysis showed that an ensemble of 50 ANN slightly outperformed the RF. In other words, Bayesian regularization and Levenberg-Marquardt algorithm appeared to be less biased and more precise, as compared with the RF.

Analyzing the results of the prediction, it was found out that the depth of cracks was more difficult to predict accurately than its location. The data set of eight natural frequency parameters produced more accurate predictions on the crack depth; meanwhile, the data set of eight Haar wavelet coefficients produced more precise predictions on the crack locations.

Acknowledgements. Financial support from the Estonian Research Council under Grant IUT34-4 is gratefully acknowledged. Special acknowledgements go to the referee and the editor for the comments and guidelines.

\section{References}

[1] M. Attar, A transfer matrix method for free vibration analysis and crack identification of stepped beams with multiple edge cracks and different boundary conditions, Internat. J. Mech. Sci 57(1) (2012), 19-33.

[2] M. H. Beale, M. T. Hagan, and H. B. Demuth, Neural Network ToolboxTM User's Guide, in: The MathWorks, Inc., Massachusetts, 2016, pp. 131, 914.

[3] L. Breiman, Random forests, Machine Learning 45(1) (2001), 5-32.

[4] S. Caddemi and I. Caliò, Exact solution of the multi-cracked Euler-Bernoulli column, Internat. J. Solids Structures 45(5) (2008), 1332-1351.

[5] S. Caddemi and A. Morassi, Multi-cracked Euler--Bernoulli beams: Mathematical modeling and exact solutions, Internat. J. Solids Structures 50(6) (2013), 944-956.

[6] A. D. Dimarogonas, S. A. Paipetis, and T. G. Chondros, Analytical Methods in Rotor Dynamics, Elsevier Applied Science, 1986. 
[7] U. Eroglu and E. Tufekci, Exact solution based finite element formulation of cracked beams for crack detection, Internat. J. Solids Structures 96 (2016), 240-253.

[8] F. A. Farret, M. Simoes, and D. Brandao, Electronic Instrumentation for Distributed Generation and Power Processes, CRC Press, Boca Raton FL, 2017.

[9] L. J. Hadjileontiadis, E. Douka, and A. Trochidis, Fractal dimension analysis for crack identification in beam structures, Mech. Syst. Signal Process. 19(3) (2005), 659-674.

[10] S. J. S. Hakim, A. H. Razak, and S. A. Ravanfar, Fault diagnosis on beam-like structures from modal parameters using artificial neural networks, Measurement: Journal of the International Measurement Confederation. 76 (2015), 45-61.

[11] Y. He, D. Guo, and F. Chu, Using genetic algorithms and finite element methods to detect shaft crack for rotor-bearing system, Math. Comp. Simulation 57(1-2) (2001), 95-108.

[12] P. Konar and P. Chattopadhyay, Bearing fault detection of induction motor using wavelet and support vector machines (SVMs), Appl. Soft Comput. 11(6) (2011), 42034211.

[13] M. A. Mahmoud and M. A. A. Kiefa, Neural network solution of the inverse vibration problem, NDT \& Internat. 32(2) (1999), 91-99.

[14] J. Majak, M. Pohlak, K. Karjust, M. Eerme, J. Kurnitski, and B. S. Shvartsman, New higher order Haar wavelet method: Application to FGM structures, Compos. Structures 201 (2018), 72-78.

[15] B. P. Nandwana and S. K. Maiti, Modelling of vibration of beam in presence of inclined edge or internal crack for its possible detection based on frequency measurements, Engrg. Fracture Mech. 58(3) (1997), 193-205.

[16] N. Papaeconomou and A. Dimarogonas, Vibration of cracked beams, Comput. Mech. 5(2) (1989), 88-94.

[17] P. F. Rizos, N. Aspragathos, and A. D. Dimarogonas, Identification of crack location and magnitude in a cantilever beam from the vibration modes, J. Sound Vibration 138(3) (1990), 381-388.

[18] M. B. Rosales, C. P. Filipich, and F. S. Buezas, Crack detection in beam-like structures, Engrg. Structures 31(10) (2009), 2257-2264.

[19] M. Rucka and K. Wilde, Application of continuous wavelet transform in vibration based damage detection method for beams and plates, J. Sound Vibration 297(3) (2006), 536-550.

[20] M. Rucka and K. Wilde, Crack identification using wavelets on experimental static deflection profiles, Engrg. Structures 28(2) (2006), 279-288.

[21] M. K. Sutar, S. Pattnaik, and J. Rana, Neural based controller for smart detection of crack in cracked cantilever beam, in: 4th International Conference on Materials Processing and Characterzation 2(4-5) (2015), pp. 2648-2653.

[22] J. M. Twomey and A. E. Smith, Performance measures, consistency, and power for artificial neural network models, Math. Comput. Modelling 21(1) (1995), 243-258.

[23] K. Worden, W. J. Staszewski, and J. J. Hensman, Natural computing for mechanical systems research: A tutorial overview, Mech. Systems Signal Process. 25(1) (2011), $4-111$.

[24] Y. J. Yan, L. Cheng, Z. Y. Wu, and L. H. Yam, Development in vibration-based structural damage detection technique, Mech. Systems Signal Process. 21(5) (2007), 21982211.

Institute of Computer Science, University of Tartu, 50409 Tartu, Estonia

E-mail address: helle.hein@ut.ee

E-mail address: ljubov.jaanuska@ut.ee 